\title{
Staphylococcus aureus bacteremias following liver transplantation: a clinical analysis of 20 cases
}

\author{
This article was published in the following Dove Press journal: \\ Therapeutics and Clinical Risk Management \\ 12 June 2015 \\ Number of times this article has been viewed
}

\author{
Jiandang Zhou', \\ Hui Huang ${ }^{3}$ \\ Shan $\mathrm{Liu}^{4}$ \\ Ping $\mathrm{Yu}^{2}$ \\ Qiquan $\mathrm{Wan}^{5}$
}

'Department of Clinical Laboratory, the Third Xiangya Hospital of Central South University, ${ }^{2}$ Department of Immunology, Xiangya School of Medicine, Central South University, ${ }^{3}$ Nursing Department, the Third Xiangya Hospital of Central South University, Changsha, Hunan, People's Republic of China; ${ }^{4}$ Adelphi University College of Nursing and Public Health, New York, NY, USA; ${ }^{5}$ Department of Transplant Surgery, the Third Xiangya Hospital of Central South University, Changsha, Hunan, People's Republic of China
Correspondence: Qiquan Wan Department of Transplant Surgery, the Third Xiangya Hospital of Central South University, No 138 Tongzipo Road,

Changsha 4I00I3, Hunan, People's Republic of China

$\mathrm{Tel}+8673|886| 8312$

Fax $+8673|886| 8312$

Email I3548685542@I63.com
Background: To describe the incidence, clinical characteristics, and outcomes of Staphylococcus aureus bacteremia after liver transplantation and investigate the drug resistance of $S$. aureus to frequently used antibiotics to provide evidence for clinical prevention and therapy.

Materials and methods: In a double-center retrospective study, blood cultures positive for $S$. aureus were obtained from January 1, 2001 to December 31, 2014. The BACTEC 9120 blood culture system and the Vitek-2 system were used to process blood samples and identify species, respectively. We also collected these patients' data to confirm clinical and laboratory characteristics.

Results: Twenty of 275 (7.3\%) liver recipients developed $S$. aureus bacteremia during the study period. The median time to the onset of $S$. aureus bacteremias was 6 days after liver transplantation and all episodes of bacteremias were early onset. The lung was the most common source of primary infection, followed by the intra-abdominal/biliary tract. A total of nine (45\%) liver recipients died due to $S$. aureus bacteremias. Of these 20 S. aureus cases, $80 \%$ were methicillin-resistant. S. aureus was highly resistant to erythromycin and penicillin (resistance rate $>90 \%$ ). No $S$. aureus resistant to glycopeptides and oxazolidone antibiotics was observed. There were seven (35\%) liver recipients with an inappropriate antibiotic therapy. Between the periods of 2001-2007 and 2008-2014, the distribution of methicillin-resistant S. aureus was not significantly different $(P=1.000)$. Pneumonia as a predominant primary source, a high body temperature, abnormal blood pressure, and decreased platelets, which occurred in the early period after liver transplantation, as well as high morbidity and mortality, were the main characteristics of $S$. aureus bacteremias.

Conclusion: $S$. aureus led to severe bacteremias in liver recipients, with high morbidity and mortality, and the majority of them comprised methicillin-resistant S. aureus.

Keywords: liver transplantation, Staphylococcus aureus, bacteremia, drug resistance

\section{Introduction}

Staphylococcus aureus bacteremia is an important issue complicating the clinical course of liver recipients and has been associated with significant morbidity and mortality, as shown in previous studies with a reported incidence rate of $15 \%-66 \%$ and a mortality rate of $21 \% .{ }^{1-3}$ Our previous study revealed that $S$. aureus was responsible for $26 \%$ of all pathogens causing bacteremias among liver transplant recipients. ${ }^{4}$

Some researchers have reported a high incidence of methicillin-resistant S. aureus (MRSA) in liver transplant recipients. ${ }^{3,5,6}$ The increasing antibiotic-resistant $S$. aureus has emerged as a pivotal factor that influences the prognosis and survival of liver transplant recipients. Herein, we aimed to describe the incidence, clinical and laboratory characteristics, and outcomes of $S$. aureus bacteremias after liver transplantation and to investigate the drug resistance of $S$. aureus to frequently used antibiotics to provide evidence for clinical prevention and therapy. 


\section{Materials and methods}

\section{Study population and clinical isolates}

This study was conducted both at the Third Xiangya Hospital, an 1,800-bed teaching hospital, affiliated with the Central South University, Changsha, People's Republic of China, with an active transplantation program (annual average of 30 liver transplants and 150 kidney transplants) and Zhongnan Hospital, a 1,200-bed teaching hospital, affiliated with Wuhan University, Wuhan, People's Republic of China, with an active transplantation program (annual average of 40 liver transplants and 100 kidney transplants). Liver recipients with an episode of $S$. aureus bacteremia, between January 1, 2001 and December 31, 2014, were identified from the microbiology laboratory database. Maintenance immunosuppression was based on calcineurin inhibitor (cyclosporine or tacrolimus) and corticosteroids, with or without mycophenolate mofetil. Cyclosporine was administrated to three patients, and tacrolimus was prescribed in the remaining 17 patients. Doses of cyclosporine were adjusted to obtain trough plasma levels of $200-300 \mathrm{ng} / \mathrm{mL}$ during the first month and 100-150 ng/mL later on. The initial dose of oral tacrolimus was $0.05 \mathrm{mg} / \mathrm{kg}$ twice a day and adjusted to achieve a targeted trough level of $8-10 \mathrm{ng} / \mathrm{mL}$ for the first 3 months and 5-6 ng/mL thereafter. Mycophenolate mofetil was prescribed in four patients at a dose of $1 \mathrm{~g}$ per day, and the dose was adjusted in response to adverse events. All patients received steroids at a dose of $500 \mathrm{mg}$ /day via intravenous on transplant day 0 then tapered to $5 \mathrm{mg}$ per day, leading to steroid-free maintenance after 6 months.

The prophylactic antibiotics used during liver transplantation included second- or third-generation cephalosporins, semisynthetic penicillins/beta-lactamase inhibitors, and carbapenems according to susceptibility patterns of the bacteria isolated before operation. Clinical characteristics including age, sex, body temperature at bacteremia onset, time of bacteremia onset, nosocomial origin, site of primary infection, inappropriate antimicrobial use, the number of MRSA cases, septic shock, and laboratory data were collected for the analysis. The laboratory data were collected in the first 24 hours after the blood culture was drawn, including serum creatinine levels, serum albumin levels, white blood cell count, platelet count, and lymphocyte count. The present study was approved by the two hospitals' ethics committees.

\section{Definitions}

Bacteremias were defined according to Centers for Disease Control and Prevention criteria. ${ }^{7}$ An episode of bacteremia was defined by at least one positive blood culture with
S. aureus in the presence of clinical signs of infection. The source of bacteremia was determined if focal signs or symptoms of infection were present, and the same microorganism was found in the blood isolate, as well the infected site. ${ }^{8}$ Appropriate antimicrobial therapy was defined as the use of an in vitro susceptible antibiotic against $S$. aureus within 24 hours of the first positive blood culture. ${ }^{9}$ Nosocomial bacteremia was defined as a positive blood culture obtained from patients who had been hospitalized for 48 hours or longer. ${ }^{10}$ We identified a microorganism as the same microorganism when the same biotype and the same antibiotype were identified in both the blood and the primary infected site, without molecular analysis being performed. Fisher's exact test was used to compare the distribution of MRSA between the period of 2001-2007 and 2008-2014, and a two-sided value of $P<0.05$ was considered significant.

\section{Microbiologic studies}

A $10 \mathrm{~mL}$ blood sample was injected into each bottle of a set of aerobic and anaerobic blood culture bottles, and they were immediately transported to the clinical microbiology laboratory for bacterial culture and identification. The BACTEC ${ }^{\text {TM }}$ 9120 blood culture system (BD, Franklin Lakes, NJ, USA) and the VITEK ${ }^{\circledR} 2$ system (bioMérieux, Craponne, France) were used to process blood samples and identified species, respectively. The Kirby-Bauer disk diffusion method was used for determining antimicrobial susceptibility and agar dilution for measuring the minimum inhibitory concentration. For amikacin, levofloxacin, trimethoprim-sulfamethoxazole, penicillin, erythromycin, clindamycin, oxacillin, and rifampicin, the Kirby-Bauer disk diffusion method was used. The oxacillin disk was a reference method for the identification of MRSA strains. Resistance of vancomycin, teicoplanin, and linezolid was defined by minimum inhibitory concentration determination. According to Zhang, ${ }^{11}$ the agar dilution of vancomycin and teicoplanin was applied.

S. aureus 25923, which belonged to American Type Culture Collection strains (Manassas, VA, USA), was used to perform quality control. The results were interpreted according to the National Committee for Clinical Laboratory Standards manual, ${ }^{12}$ which was suitable for 2001-2003 and the Clinical Laboratory Standards Institute criteria, ${ }^{13}$ which was suitable for 2004-2014. Intermediate susceptibility to the antibiotics was classified as resistance.

\section{Antibiotics for bacteria}

Amikacin, levofloxacin, trimethoprim-sulfamethoxazole, penicillin, erythromycin, clindamycin, oxacillin, rifampicin, 
vancomycin, teicoplanin, and linezolid were products of Oxoid Ltd (Hampshire, UK). The powder of vancomycin and teicoplanin was also obtained from Oxoid Ltd (Hampshire, UK).

\section{Results}

\section{Clinical manifestation}

Twenty of 275 (7.3\%) liver recipients developed S. aureus bacteremias from January 1, 2001 to December 31, 2014, involving 19 (95\%) male and one (5\%) female patients aged 15-67 years (the average age was $42.7 \pm 14.5$ years). A total of $19(95 \%)$ patients had central vein catheters at the onset of $S$. aureus bacteremias. Sixteen patients experienced extensive exposure to broad-spectrum antibiotics in the pretransplant phase. The median time of hospitalization prior to transplantation was 25 days (range: $2-121$ days). We elaborated upon the clinical manifestation of liver recipients with $S$. aureus bacteremias as follows. First, there was fever and abnormal blood pressure. The majority of recipients had a fever and there were $12(60 \%)$ episodes of bacteremias accompanied by a body temperature of $38^{\circ} \mathrm{C}$ or greater. A total of nine (45\%) liver recipients developed septic shock at the onset of S. aureus bacteremias. Second, 20 (100\%) episodes of $S$. aureus bacteremias were nosocomial infection. Third, the onset occurred in the early period after liver transplantation. The median time to the onset of $S$. aureus bacteremias after liver transplantation was 6 days (interquartile range: 4-9 days) and all bacteremias were early onset (within 1 month of transplantation). Fourth, 17 (85\%) episodes of bacteremias were secondary to other infections. The most common site of primary infection was the lung (55\%; number $[\mathrm{n}]=11)$, followed by the intra-abdominal/biliary tract $(30 \%$; $n=6)$ and unknown sources $(15 \% ; n=3)$. Fifth, there was a high rate of inappropriate antibiotic therapy. Among these 20 liver recipients, two, three, and eight received levofloxacin, linezolid, and glycopeptide antibiotics, respectively, which had in vitro activity against $S$. aureus at the onset or within 1 day of $S$. aureus bacteremias. Ceftazidime, cefepime, and meropenem, all of which did not have in vitro activity against $S$. aureus, were prescribed in two patients each at the onset or within 1 day of $S$. aureus bacteremias, and piperacillintazobactam was prescribed in one patient with inappropriate antimicrobial use. Finally, there was high mortality. A total of nine (45\%) liver recipients died within 1 month after they were diagnosed with $S$. aureus bacteremias. Among these 20 patients with $S$. aureus bacteremias, 13 patients experienced polymicrobial bacteremias and seven patients who expired belonged to this group. The clinical and demographic characteristics of 20 liver transplant patients with $S$. aureus bacteremias are outlined in Table 1 .

\section{Laboratory data}

White blood cell count increased obviously. The amount of white blood cells in $40 \%$ of patients $(8 / 20)$ with $S$. aureus bacteremias was more than $15 \times 10^{9} / \mathrm{L}$, and the highest could reach $32.5 \times 10^{9} / \mathrm{L}$. Platelets dropped markedly. The amount of platelets in $50 \%$ of patients with bacteremia was less than $50 \times 10^{9} / \mathrm{L}$, and it could decrease to $3 \times 10^{9} / \mathrm{L}$. Lymphocyte count decreased obviously. The amount of lymphocyte count in six $(30 \%)$ patients was less than $300 / \mathrm{mm}^{3}$, and the lowest could reach $0.05 \times 10^{9} / \mathrm{L}$. Procalcitonin elevated highly. The highest serum level of procalcitonin was more than $10 \mathrm{ng} / \mathrm{mL}$. The laboratory data of these 20 liver recipients with $S$. aureus bacteremias are also illustrated in Table 1.

\section{Drug resistance to $S$. aureus}

Of these $20 \mathrm{~S}$. aureus cases, MRSA (80\%; n=16) was the predominant bacterium. S. aureus was highly resistant to erythromycin and penicillin (resistance rate $>90 \%$ ).

Table I Demographic, laboratory, and clinical characteristics of 20 liver recipients with Staphylococcus aureus bacteremias

\begin{tabular}{|c|c|}
\hline Characteristics & Value \\
\hline Age, mean years \pm standard deviation & $42.7 \pm 14.5$ \\
\hline Sex, number of males/number of females & $19 / 1$ \\
\hline Temperature of $38^{\circ} \mathrm{C}$ or greater & $12 / 20$ \\
\hline Nosocomial origin & $20 / 20$ \\
\hline Inappropriate antimicrobial use & $7 / 20$ \\
\hline Septic shock & $9 / 20$ \\
\hline \multicolumn{2}{|l|}{ Site of primary infection, number of cases (\%) } \\
\hline Lung & II (55) \\
\hline Intra-abdominal/biliary & $6(30)$ \\
\hline Unknown & $3(15)$ \\
\hline \multicolumn{2}{|l|}{$\begin{array}{l}\text { Time of bacteremia onset (post-transplant), } \\
\text { number of cases (\%) }\end{array}$} \\
\hline$\leq 2$ weeks & $16(80)$ \\
\hline$>2$ weeks & $4(20)$ \\
\hline \multicolumn{2}{|l|}{ MRSA, number of cases (\%) } \\
\hline Yes & $16(80)$ \\
\hline No & $4(20)$ \\
\hline \multicolumn{2}{|l|}{ Type of organisms, number of cases (\%) } \\
\hline Monomicrobial & $7(35)$ \\
\hline Polymicrobial & $13(65)$ \\
\hline \multicolumn{2}{|l|}{ Laboratory variables, number of cases (\%) } \\
\hline Platelet count $<50,000 / \mathrm{mm}^{3}$ & $10(50)$ \\
\hline Lymphocyte count $<300 / \mathrm{mm}^{3}$ & $6(30)$ \\
\hline Albumin level $<30 \mathrm{mg} / \mathrm{dL}$ & $3(15)$ \\
\hline WBC count $>15,000 / \mathrm{mm}^{3}$ & $8(40)$ \\
\hline Creatinine level $>1.5 \mathrm{mg} / \mathrm{dL}$ & $5(25)$ \\
\hline Related mortality & $9(45)$ \\
\hline
\end{tabular}

Abbreviations: MRSA, methicillin-resistant Staphylococcus aureus; WBC, white blood cell. 
Table 2 Resistance of $S$. aureus to eleven antibiotics

\begin{tabular}{|c|c|c|c|c|c|c|c|c|c|c|c|}
\hline Antibiotics & ERY & CC & LVF & SXT & AN & PEN & RA & ox & VAN & TEC & LZD \\
\hline S. aureus (20) & $18(90)$ & $14(70)$ & $10(50)$ & $15(75)$ & $6(30)$ & $19(95)$ & $2(10)$ & $16(80)$ & $0(0)$ & $0(0)$ & $0(0)$ \\
\hline
\end{tabular}

Notes: Data presented as number of cases (\%).

Abbreviations: S. aureus, Staphylococcus aureus; ERY, erythromycin; CC, clindamycin; LVF, levofloxacin; SXT, trimethoprim-sulfamethoxazole; AN, amikacin; PEN, penicillin; RA, rifampicin; OX, oxacillin; VAN, vancomycin; TEC, teicoplanin; LZD, linezolid.

The D-test-positive rate of inducible resistance to clindamycin was $50 \%$ in four cases of $S$. aureus, which were erythromycin resistant and clindamycin sensitive by individual disk diffusion test. None of the $S$. aureus strains was resistant to glycopeptides and oxazolidone antibiotics. All but two S. aureus strains were susceptible to rifampicin. The drug resistance of $S$. aureus to the eleven antibiotics investigated could be seen in Table 2. No significant differences in the frequency of MRSA were observed between 2001-2007 and 2008-2014 (80\% [12/15] versus $80 \%$ [4/5], respectively; $P=1.000)$.

\section{Discussion}

S. aureus bacteremias, especially MRSA bacteremia, are still a cause of morbidity and mortality in liver recipients so far. In the present study, all episodes of $S$. aureus bacteremias were early onset (within 1 month after liver transplantation), which was consistent with the findings in a previous study, ${ }^{14}$ which reported that the majority of the Gram-positive bacteremias occurred within 30 days after liver transplantation.

In the present study, the drug susceptibility test showed that the resistance rate of $S$. aureus to the eleven antibiotics investigated was relatively low, although MRSA was the predominant bacterium. S. aureus was highly susceptible to glycopeptides and oxazolidinone antibiotics in our present study.

A high incidence $(80 \%)$ of MRSA was noted in this study when compared with methicillin-susceptible $S$. aureus, in line with other studies which confirmed that, among bacteremic liver transplantation, more than $90 \%$ of $S$. aureus strains were MRSA. ${ }^{15,16}$ This is most likely explained by the presence of known risk factors related to MRSA, such as intravenous catheters, hospitalization, and antibiotic exposure, which are also present in our study group. ${ }^{17,18}$ MRSA represents a serious problem among bacteremic liver recipients. ${ }^{14,19,20}$

We found that $S$. aureus strains causing bacteremias after liver transplantation had low drug resistance $(\leq 50 \%)$ to the majority (6/11) of antibiotics investigated. There were no episodes of glycopeptide- or oxazolidinone-resistant $S$. aureus. All but two episodes were susceptible to rifampicin. The possible explanations to this finding include evolving trends of Gram-positive bacteria toward multiple-antibiotic-resistant bacteria are slowly, when compared with Gram-negative bacteria. ${ }^{21}$ Physicians can choose glycopeptides alone or oxazolidinone antibiotics alone for empirical therapy in patients that are highly suspected to have $S$. aureus bacteremia when awaiting confirmation of the bacterial identity and susceptibility pattern. It is notable that combination therapy for MRSA bacteremia appears to be no more effective than vancomycin monotherapy. Adding rifampicin to vancomycin offers no meaningful benefit and may confer harm for treating MRSA bacteremia. ${ }^{22}$ Furthermore, there is no clinical rationale to refer to rifampicin after liver transplantation, as it is known that it may eliminate calcineurin inhibitors.

\section{Conclusion}

In conclusion, $S$. aureus led to severe bacteremias in liver recipients, with high morbidity and mortality. Although the majority of the strains comprised MRSA, S. aureus had relatively low drug resistance to the majority of antibiotics investigated.

\section{Acknowledgment}

This work was support by grant 2013TT1001 from the key project of the Hunan Provincial Science Technology Department, People's Republic of China.

\section{Disclosure}

The authors report no conflicts of interest in this work.

\section{References}

1. Singh N, Paterson DL, Chang FY, et al. Methicillin-resistant Staphylococcus aureus: the other emerging resistant gram-positive coccus among liver transplant recipients. Clin Infect Dis. 2000;30(2): 322-327.

2. Singh N, Paterson DL, Gayowski T, Wagener MM, Marino IR. Predicting bacteremia and bacteremic mortality in liver transplant recipients. Liver Transpl. 2000;6(1):54-61.

3. Torre-Cisneros J, Herrero C, Cañas E, Reguera JM, De La Mata M, Gómez-Bravo MA. High mortality related with Staphylococcus aureus bacteremia after liver transplantation. Eur J Clin Microbiol Infect Dis. 2002;21(5):385-388.

4. Wan QQ, Ye QF, Ming YZ, Ma Y, Zhou JD, Qiao BB. The risk factors for mortality in deceased donor liver transplant recipients with bloodstream infections. Transplant Proc. 2002;45(1):305-307.

5. Malinis MF, Mawhorter SD, Jain A, Shrestha NK, Avery RK, van Duin D. Staphylococcus aureus bacteremia in solid organ transplant recipients: evidence for improved survival when compared with nontransplant patients. Transplantation. 2012;93(10):1045-1050. 
6. Bert F, Galdbart JO, Zarrouk V, et al. Association between nasal carriage of Staphylococcus aureus and infection in liver transplant recipients. Clin Infect Dis. 2000;31(5):1295-1299.

7. Garner JS, Jarvis WR, Emori TG, Horan TC, Hughes JM. CDC definitions for nosocomial infections, 1988. Am J Infect Control. 1988; 16(3):128-140.

8. Ye QF, Zhao J, Wan QQ, Qiao BB, Zhou JD. Frequency and clinical outcomes of ESKAPE bacteremia in solid organ transplantation and the risk factors for mortality. Transpl Infect Dis. 2014;16(5):767-774.

9. Wan Q, Ye Q, Zhou J. Mortality predictors of bloodstream infections in solid-organ transplant recipients. Exp Clin Transplant. 2013;11(3): 211-214.

10. Wan Q, Ye Q, Su T, Zhou J. The epidemiology and distribution of pathogens and risk factors for mortality in liver transplant recipients with Gram negative bacteremia. Hepatogastroenterology. 2014;61(134): 1730-1733.

11. Zhang XZ, editor. Contemporary Laboratory and Clinical Bacteriology. Beijing, People's Republic of China: The People's Press; 1999: 418-420.

12. National Committee for Clinical Laboratory Standards. Reference Method for Broth Dilution Antifungal Susceptibility Testing of Yeast. 2nd ed. Approved Standard M27-A2. Wayne, PA: National Committee for Clinical Laboratory Standards; 2002.

13. Clinical and Laboratory Standards Institute. Performance Standards for Antimicrobial Susceptibility Testing. 22nd Informational Supplement. Wayne, PA: Clinical and Laboratory Standards Institute; 2012.

14. Lee SO, Kang SH, Abdel-Massih RC, Brown RA, Razonable RR. Spectrum of early-onset and late-onset bacteremias after liver transplantation: implications for management. Liver Transpl. 2011;17(6):733-741.
15. Kim HK, Park YK, Wang HJ, et al. Epidemiology and clinical features of post-transplant bloodstream infection: an analysis of 222 consecutive liver transplant recipients. Infect Chemother. 2013;45(3):315-324.

16. Shi SH, Kong HS, Jia CK, et al. Coagulase-negative staphylococcus and enterococcus as predominant pathogens in liver transplant recipients with Gram-positive coccal bacteremia. Chin Med J (Engl). 2010; 123(15):1983-1988.

17. Lodise TP, McKinnon PS, Rybak M. Prediction model to identify patients with Staphylococcus aureus bacteremia at risk for methicillin resistance. Infect Control Hosp Epidemiol. 2003;24(9):655-661.

18. Pujol M, Peña C, Pallares R, Ayats J, Ariza J, Gudiol F. Risk factors for nosocomial bacteremia due to methicillin-resistant Staphylococcus aureus. Eur J Clin Microbiol Infect Dis. 1994;13(1):96-102.

19. Iida T, Kaido T, Yagi S, et al. Posttransplant bacteremia in adult living donor liver transplant recipients. Liver Transpl. 2010;16(12): 1379-1385.

20. Bert F, Larroque B, Paugam-Burtz C, et al. Microbial epidemiology and outcome of bloodstream infections in liver transplant recipients: an analysis of 259 episodes. Liver Transpl. 2010;16(3):393-401.

21. Singh N, Gayowski T, Rihs JD, Wagener MM, Marino IR. Evolving trends in multiple-antibiotic-resistant bacteria in liver transplant recipients: a longitudinal study of antimicrobial susceptibility patterns. Liver Transpl. 2001;7(1):22-26.

22. Levine DP, Fromm BS, Reddy BR. Slow response to vancomycin or vancomycin plus rifampin in methicillin-resistant Staphylococcus aureus endocarditis. Ann Intern Med. 1991;115(9):674-680.
Therapeutics and Clinical Risk Management

\section{Publish your work in this journal}

Therapeutics and Clinical Risk Management is an international, peerreviewed journal of clinical therapeutics and risk management, focusing on concise rapid reporting of clinical studies in all therapeutic areas, outcomes, safety, and programs for the effective, safe, and sustained use of medicines. This journal is indexed on PubMed Central, CAS,

\section{Dovepress}

EMBase, Scopus and the Elsevier Bibliographic databases. The manuscript management system is completely online and includes a very quick and fair peer-review system, which is all easy to use. Visit http://www.dovepress.com/testimonials.php to read real quotes from published authors.

Submit your manuscript here: http://www.dovepress.com/therapeutics-and-clinical-risk-management-journal 